\title{
Etude des contraintes de croissance III - Eucalyptus delegatensis et Eucalyptus nitens : Influence de la sylviculture et de la station
}

\author{
J.C. FERRAND \\ I.N.R.A., Station de Recherches sur la Qualité des Bois, \\ Centre de Recherches forestières de Nancy, \\ Champenoux, F 54280 Seichamps
}

\begin{abstract}
Résumé
Environ 550 mesures de contraintes de croissance ont été réalisées sur Eucalyptus delegatensis et Eucalyptus nitens dans des essais d'espacements à la plantation et d'éclaircie situés à proximité de Melbourne (Victoria, Australie). Il en ressort que, au même âge, plus l'espacement entre arbres est grand, plus les contraintes sont faibles. La sylviculture est donc un moyen très efficace pour diminuer les contraintes de croissance. Elles sont aussi plus faibles là où la hauteur dominante est plus grande.
\end{abstract}

Les résutats montrent qu'il est possible de prédire quel niveau de contraintes de croissance résultera d'une sylviculture donnée dans une station donnée.

\author{
Mots-clés : Contraintes de croissance - Densité à l'hectare - Hauteur dominante \\ Espèces : Eucalyptus delegatensis - Eucalyptus nitens
}

\section{Introduction}

Le premier article de cette série a montré l'intérêt d'une méthode de mesure indirecte des contraintes de croissance qui utilise des carottes de sondage. Le deuxième article a ensuite établi - entre autres - que chez le hêtre, la sylviculture est la source principale de variation de contraintes entre les peuplements : plus les arbres sont gros et espacés et moins ils ont de contraintes de croissance. L'objet de ce troisième article est de rendre compte des résultats obtenus à propos d'Eucalyptus delegatensis et d'Eucalyptus nitens lors d'une mission en Autralie dans l'aire naturelle de ces espèces.

Comme pour le hêtre, peu d'informations étaient disponibles à propos des contraintes chez les eucalyptus. Sur le plan génétique, en Australie, Waugh (1977) n'a trouvé pratiquement aucune différence entre 25 familles d' $E$. regnans récoltées à 5 altitudes sur un même versant. En revanche, MC КIMM (communication person- 

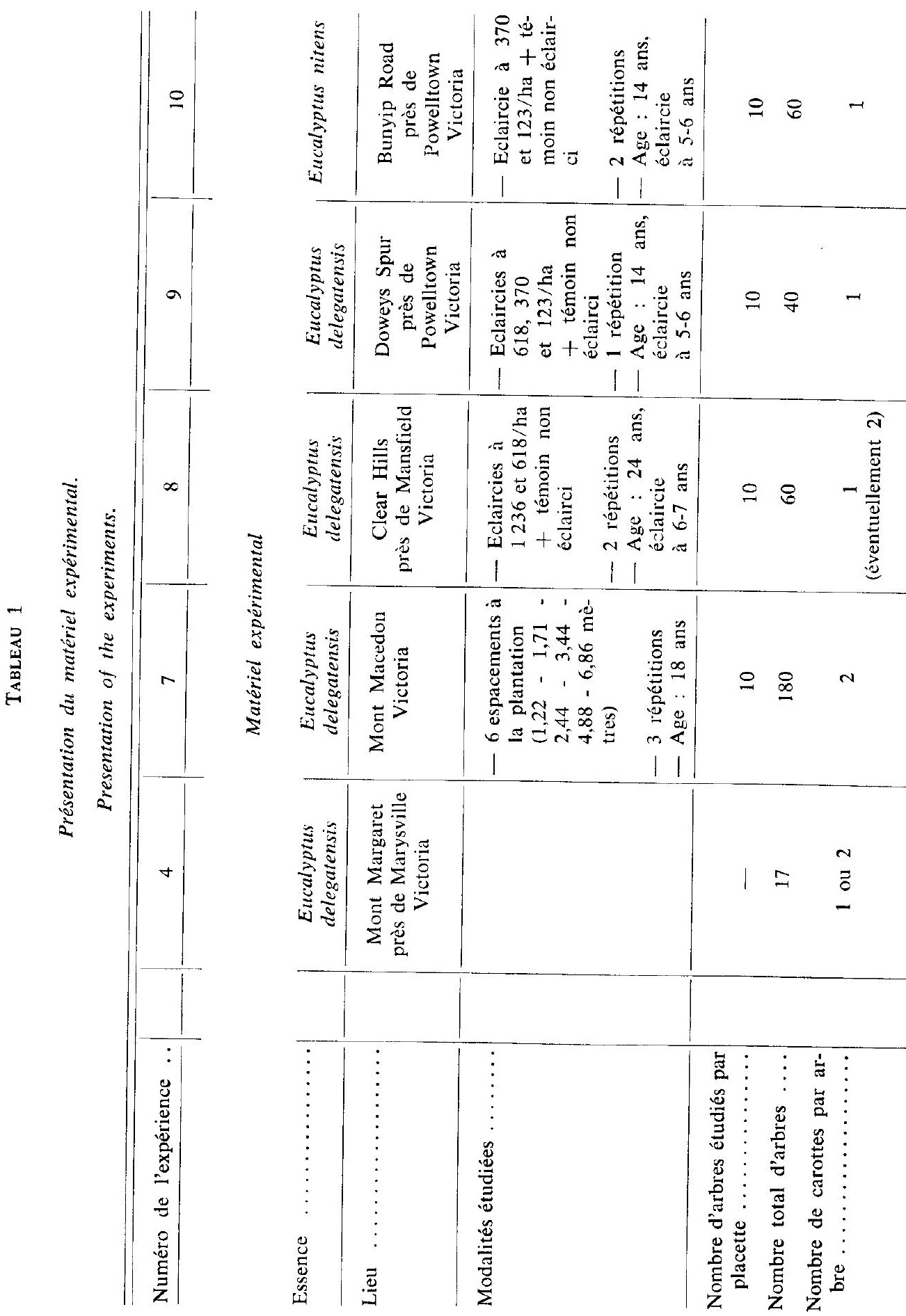
$8-\frac{5}{5}$

$\mathcal{F}=\stackrel{\Xi}{\Xi}$

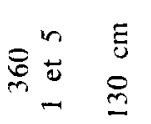

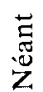

$\overrightarrow{\bar{E}}$

苛

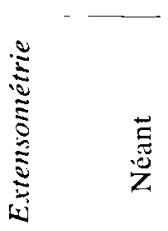

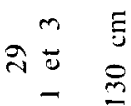

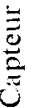
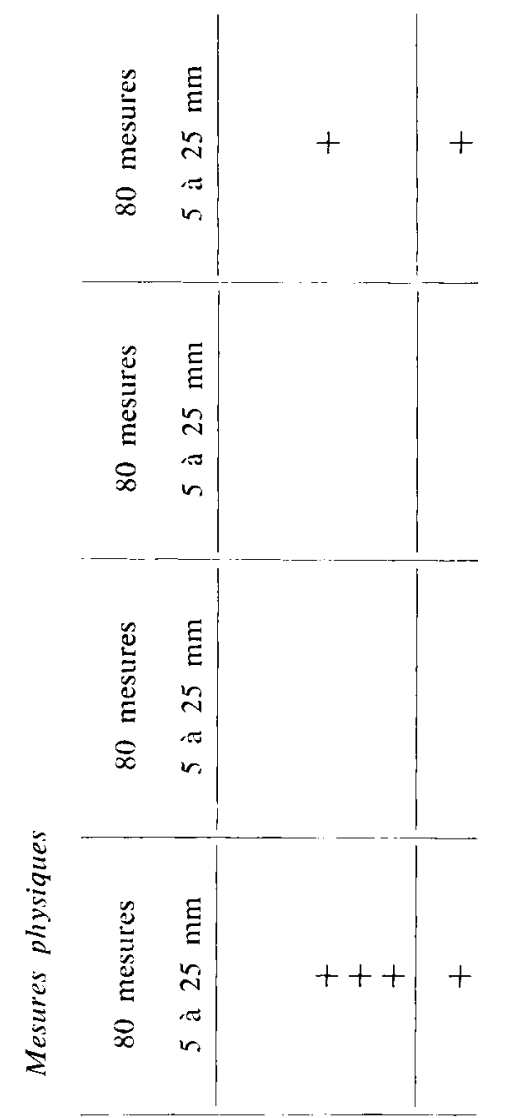

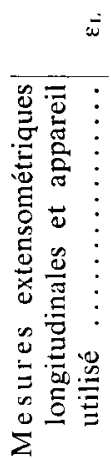

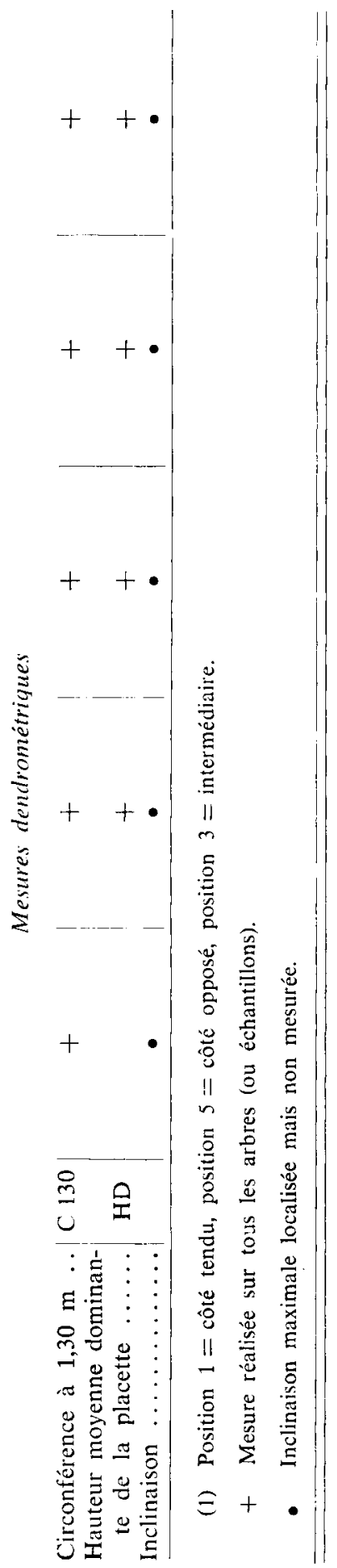

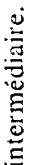


nelle, 1981) a trouvé des différences entre provenances d'E. nitens (tous deux utilisaient la méthode de mesure proposée par Nicholson, 1971). En Nouvelle-Zélande, BARR (1981) a observé lors du sciage des différences de déformations dues à la relaxation des contraintes de croissance entre lots d'E. saligna; il a même engagé une sélection récurrente à l'intérieur des meilleures populations.

Sur le plan sylvicole, pratiquement aucune information n'était disponible; de même pour l'influence de la station. C'est dans ces domaines que nous avons mené nos investigations.

\section{Matériel et méthodes}

Le matériel expérimental et les méthodes de mesure sont présentés dans un tableau $\left(\mathrm{n}^{\circ} 1\right)$, comme dans les articles précédents auxquels on peut se reporter pour la description détaillée des méthodes de mesure.

Il convient de discuter cependant la mesure des retraits et celle des hauteurs. En effet, le séchage du bois d'E. delegatensis et d'E. nitens s'accompagne de collapse. Celui-ci est variable selon les conditions du séchage et n'affecte pas l'aubier de la même manière que le cœur. Or notre zone de mesure des contraintes de croissance est en général à cheval sur l'aubier et le bois de cœur pour ces espèces à aubier peu épais. De plus, les conditions de séchage qui avaient été choisies (une semaine à l'étuve à $60^{\circ} \mathrm{C}$, une semaine en chambre pour le conditionnement à 12 p. 100 d'humidité) n'ont pas pu être respectées à la lettre, ce qui risque d'introduire une variation indésirable des valeurs constatées de ce retrait avec collapse. (Faute de temps, les carottes n'ont pu être reconditionnées et remesurées, ce qui aurait amélioré la qualité des résultats). Par conséquent ces mesures, qui n'étaient pas l'objectif principal de l'étude, doivent être considérées plus comme une indication que comme un résultat définitif sur le retrait.

D'autre part, contrairement à l'article précédent, la hauteur mesurée n’est pas celle de chaque arbre, mais la hauteur moyenne dominante par placette, telle qu'elle a été mesurée par la Forests Commission of Victoria : hauteur moyenne des 62 plus gros arbres à l'hectare. Lorsque nous emploierons l'expression anglaise "Site Index 》 (SI), celle-ci désignera la hauteur moyenne dominante à 20 ans, calculée comme suit :

$$
\text { HD }=2 \text { SI }\left(1-10^{-0.015 a x p}\right)
$$

Cette équation est celle qui permet de suivre l'évolution en hauteur des espèces telles que $E$. regnans et $E$. delegatensis. Pour une même essence, la valeur du «Site Index » caractérise la fertilité de la station. Mais si l'on change d'espèce, la valeur du «Site Index » change également; ainsi $E$. nitens, plus vigoureux, donne des S.I. plus élevés.

Comme dans les expériences précédentes, tous les arbres sondés étaient dominants. De plus, on a toujours effectué les prélèvements en fonction de la direction de l'inclinaison des arbres et plus de 90 p. 100 d'entre eux avaient une inclinaison décelable avec un simple fil à plomb. 
Les expériences réalisées avaient pour but :

- de confirmer la valeur de la méthode de mesure sur carottes de sondage dans le cas des eucalyptus,

- d'étudier l'influence de l'espacement à la plantation,

- d'étudier l'influence de différents régimes d'éclaircie.

Les résultats seront présentés dans ce même ordre. On utilisera les symboles habituels :

$$
\begin{aligned}
\text { NS : non significatif, } \\
* \text { : significatif au risque } 5 \text { p. } 100, \\
* * \text { : significatif au risque } 1 \text { p. } 100, \\
* * *: \text { significatif au risque } 1 \text { p. } 1000 .
\end{aligned}
$$

\section{Résultats pour la méthode de mesure sur carottes de sondage}

Dans le premier article de cette série (Ferrand, 1982 a) on a déjà mentionné brièvement l'expérience $\mathrm{n}^{\circ}$ 4. Elle a eu lieu à proximité de Marysville (Victoria, Australie) sur les pentes du Mont Margaret, dans l'aire naturelle d'Eucalyptus delegatensis. On a choisi des arbres dominants dans deux parcelles contiguës d'âge différent (environ 40 ans et 25 ans) et on a réalisé au même endroit une mesure de déformation et de prélèvement d'une carotte. On a trouvé que le diamètre tangentiel (TG) des carottes était corrélé avec la déformation longitudinale $\left(\varepsilon_{\mathrm{F}_{2}}\right)$ due à la relaxation des contraintes de croissance $\left(r=-0,759^{* * * * *}, 29\right.$ échantillons).

\section{La figure 1 représente cette liaison entre TG et $\varepsilon_{L}$.}

Cependant, à la différence du hêtre et du peuplier, la corrélation entre le retrait longitudinal $(\mathrm{RL})$ et $\varepsilon_{\mathrm{L}}$ est non significative. Nous avons déjà évoqué (FerRAND, $1982 \mathrm{a}$ ) la différence de structure anatomique qui existe entre les eucalyptus et les deux autres espèces en particulier pour le bois de tension, ce dernier étant responsable de la corrélation entre RL et $\varepsilon_{\mathrm{L}}$, lorsqu'elle existe. Mais une autre possibilité existe pour expliquer l'absence de corrélation rencontrée ici. C'est le collapse. En effet, le retrait longitudinal prend des valeurs très faibles (quelques millièmes) et toute interaction avec le collapse, soit par effet Poisson, soit par un changement de forme de l'échantillon peut masquer une éventuelle influence du bois de tension.

Quoi qu'il en soit, le principal est de se rappeler que, contrairement aux deux articles précédents, lorsque des valeurs de retrait longitudinal sont présentées dans les résultats, il ne faut pas les considérer comme indicatrices du niveau des contraintes de croissance. Seul le diamètre tangentiel des carottes joue ici ce rôle.

Note : La tarière utilisée pour cette expérience a dû être réaffûtée avant la réalisation des expériences suivantes à cause d'un léger défaut de fabrication. On n'a donc pas converti les valeurs de TG données dans les paragraphes suivants en valeur de $\varepsilon_{\mathrm{r}_{i}}$. 


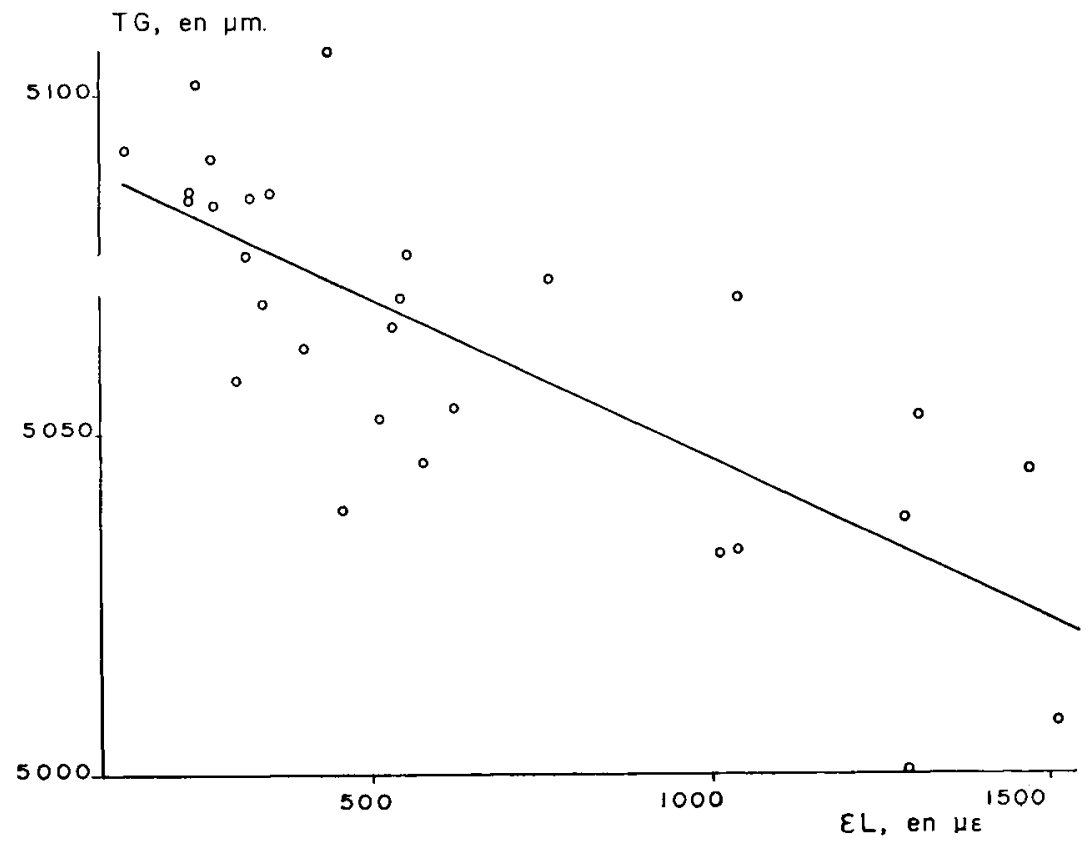

FiG. 1

Expérience $n^{\prime \prime} 4$ : Liaison entre le diamètre tangentiel des carottes (TG) et la déformation potentielle longitudinale $\left(\varepsilon_{L}\right): r=-0,759 * *$.

Experiment no. 4 : Relationship between tangential diameter of the increment cores $(T G)$ and longitudinal growth strain $\left(\varepsilon_{L}\right): r=-0.759^{*} * *$.

\section{Résultats de l'expérience d'espacement à la plantation de Mont Macedon}

L'expérience $\mathrm{n}^{\circ} 7$ est la plus importante de cette étude. Elle a été réalisée dans un essai d'espacement planté en 1963, qui comprend 3 répétitions de 6 espacements. Ceux-ci sont choisis de telle sorte que la surface disponible par arbre double à chaque fois et vont de $1,22 \mathrm{~m} \times 1,22 \mathrm{~m}$ (4 pieds $\times 4$ pieds) à $6,86 \mathrm{~m} \times 6,86 \mathrm{~m}(22,5$ pieds $\times 22,5$ pieds). Les placettes sont carrées et regroupent $7 \times 7=49$ arbres. Il y a au moins deux rangées supplémentaires au même espacement autour de chaque placette (plus de deux pour les faibles espacements). Pour ne pas endommager les arbres du peuplement principal, les carottes ont été prélevées dans la première de ces rangées supplémentaires, à raison de 10 arbres dominants par placette et deux carottes par arbre, l'une du côté tendu, l'autre du côté opposé (cf. tableau 1).

Mont Macedon est une montagne qui s'élève de manière abrupte d'environ 600 mètres au-dessus des plateaux environnants, à une cinquantainc de kilomètres au Nord de Melbourne. 
Les trois répétitions se répartissent ainsi : deux près du sommet de la montagne (environ $975 \mathrm{~m}$ d'altitude) et une moins de $100 \mathrm{~m}$ en contrebas, en bas d'une petite pente. Les arbres de la troisième répétition sont beaucoup plus grands que les autres; comme une seule et même source de graines a été utilisée, c'est donc que la «fertilité » de la station est plus élevée. Cela est confirmé par la vigueur très supérieure du sous-étage. Signalons également que les placettes d'Eucalyptus regnans installées en guise de comparaison montrent une croissance médiocre et une mortalité importante, ce qui montre que l'on se trouve bien à l'étage d'E. delegatensis - appelé ici «alpine ash»— qui est juste au-dessus de celui d'E. regnans — «mountain ash». De même, la présence d'un petit peuplement d'E. pauciflora - "snow gum»tout au sommet de la montagne (un peu au-dessus de $1000 \mathrm{~m}$ daltitude) vient confirmer le fait que la plantation d'E. delegatensis est bien « en station».

\subsection{Influence de la position autour de l'arbre}

La première préoccupation a été de vérifier s:il existe bien une différence de contraintes de croissance entre côté tendu (noté 1) et côté opposé (noté 5 comme dans l'article précédent : Ferrand, 1982 b). Le tableall 2 montre que tel est bien le cas. Cependant, la différence semble beaucoup moins importante que dans le cas du hêtre. De plus, on ne constate aucune influence de l'espacement sur cette différence, alors quelle varie énormément avec la sylviculture chez le hêtre. Il y a donc là à la fois une similitude (la différence existe) et une profonde opposition avec le hêtre (elle ne dépend pas de la sylviculture).

\section{Tableau 2}

Expérience $n^{\circ} 7$ :

Influence de la position autour du tronc sur le diamètre tangentiel des carottes (TG 1 côté tendu, TG 5 côté opposé).

Experiment no. 7 :

Influence of the position around the stem on the tangential diameter of the increment cores (TG 1 upper side, TG 5 opposite side).

\begin{tabular}{c|c|c|c|c}
\hline $\begin{array}{c}\text { TG 1 } \\
\text { en } \mu \mathrm{m}\end{array}$ & $\begin{array}{c}\text { TG } 5 \\
\text { en } \mu \mathrm{m}\end{array}$ & \multicolumn{2}{|c}{ TG 1 - TG 5 en $\mu \mathrm{m}$} \\
\hline Moyenne & Moyenne & Moyenne & Variance & Test des moyennes appariées \\
\hline 5074,3 & 5081,5 & 7,2 & 245 & $6,17^{* * *}$ \\
\hline
\end{tabular}

En revanche, il est fort possible que cette dissymétrie des contraintes de croissance dépende de l'inclinaison des troncs. Malheureusement, celle-ci n'a pas été mesurée avec précision, faute de temps. 


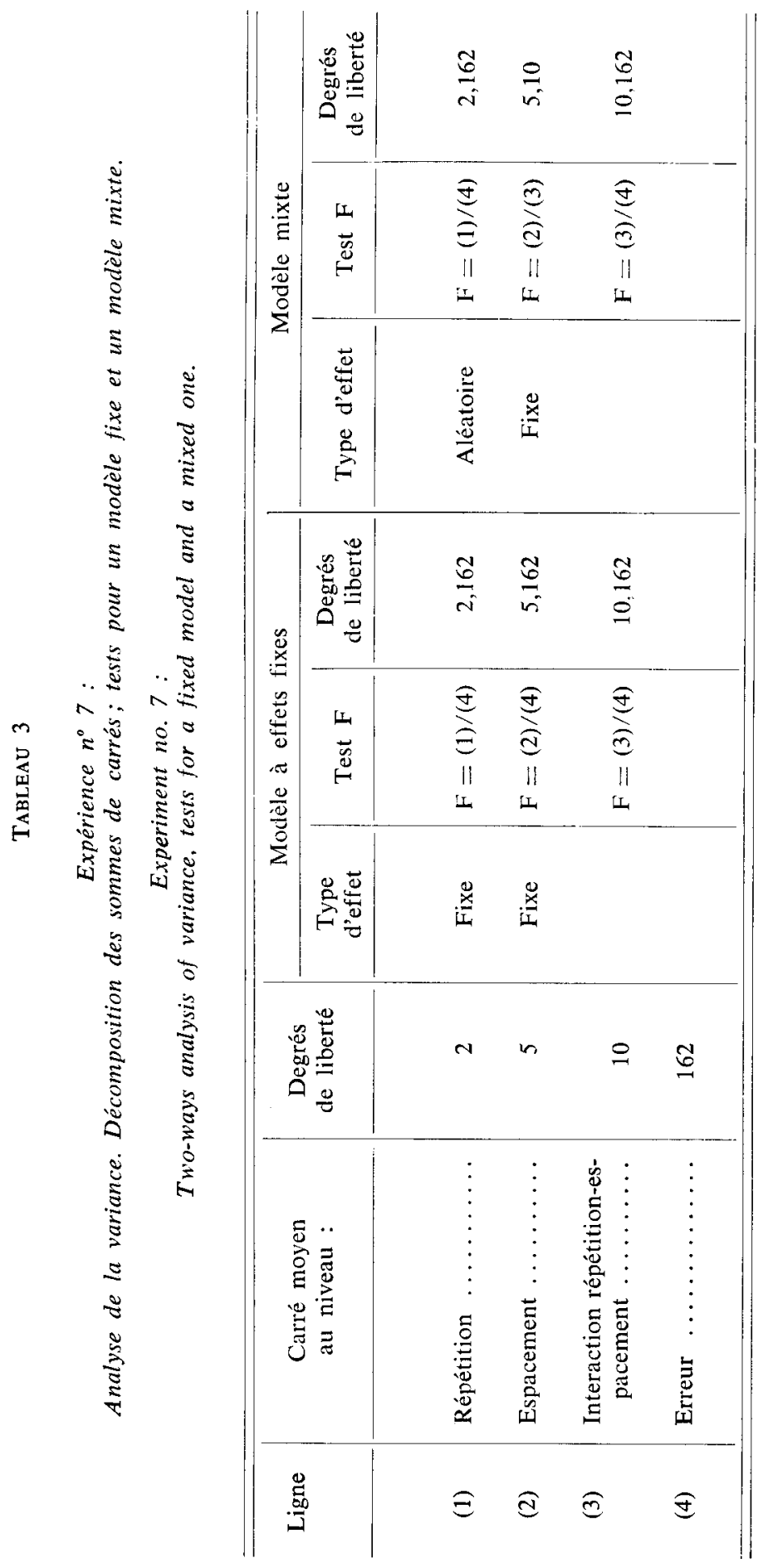




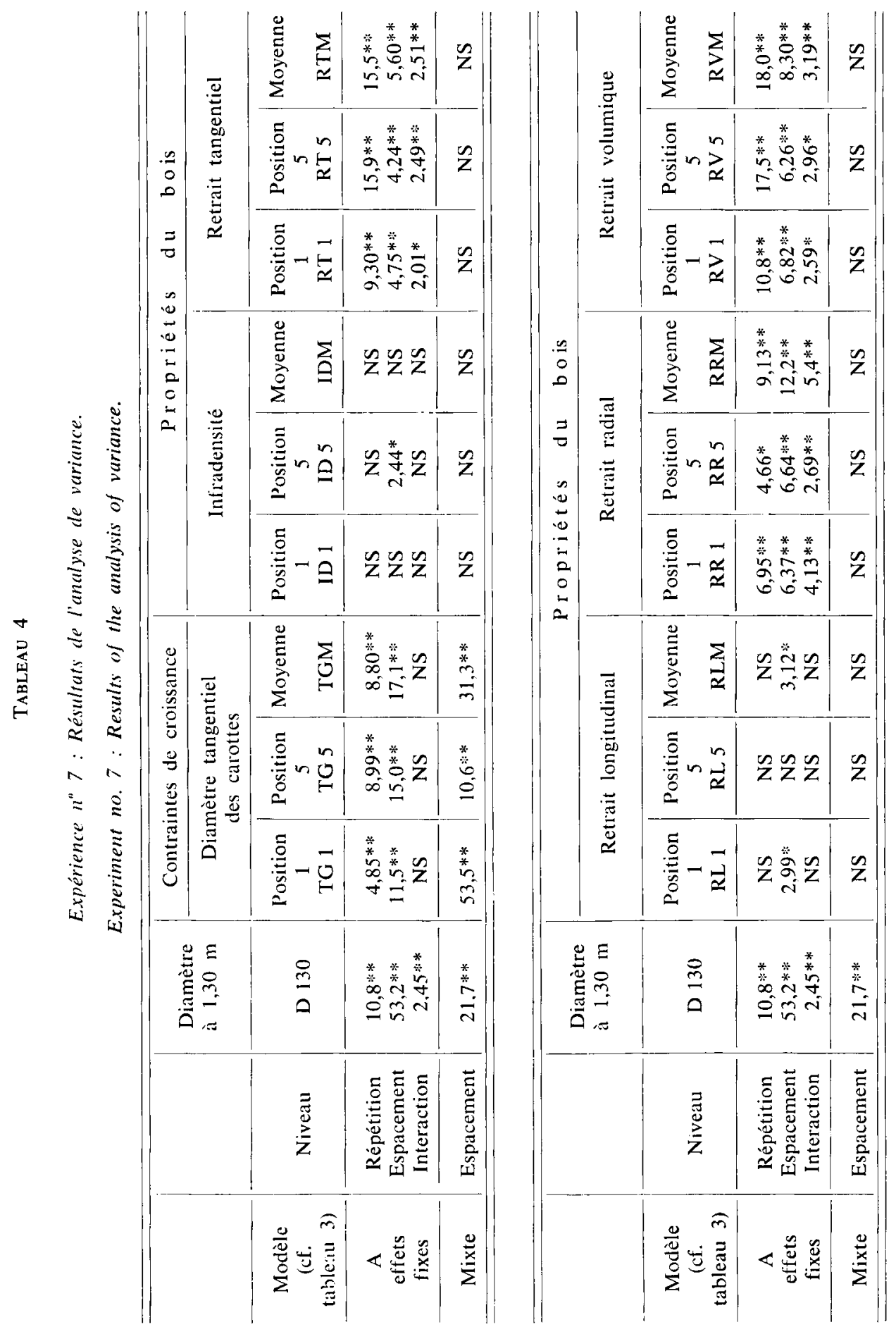



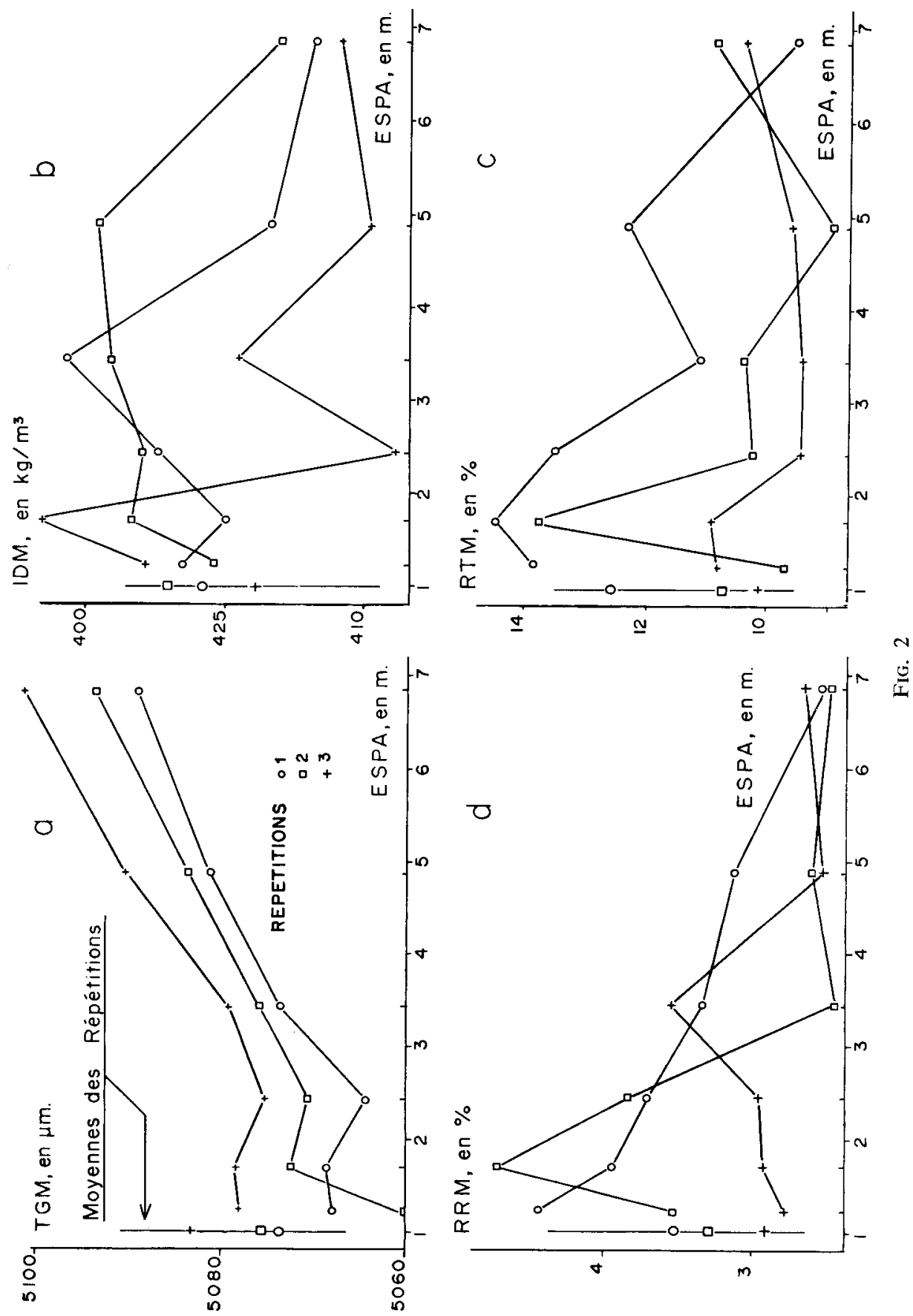


\subsection{Analyse de la variance et corrélations}

On a ensuite réalisé l’analyse de la variance pour toutes les variables mesurées. Comme le montre le tableau 3 , on a testé les effets répétition, espacement et interaction répétition-espacement. Le test $F$ a été réalisé de deux façons différentes. Dans tous les cas on a considéré l'espacement comme un facteur à effets fixes. Mais on peut considérer la répétition, c'est-à-dire la station :

- soit comme un facteur à effets fixes, auquel cas les résultats observés sur le jacteur espacement ne sont pas extrapolables dans d'autres stations (modèle à effets fixes) ;

- soit comme un facteur aléatoire, auquel cas on peut extrapoler les résultats à dautres stations dont les répétitions de l'expérience n" 7 constituent un échantillon représentatif (modèle mixte : Dagnelie, 1975).

Les résultats sont portés dans le tableau 4, pour les deux modèles du tableau 3 .

\subsection{Contraintes de croissance}

On voit que l'espacement a une grande influence sur les contraintes de croissance. Celles-ci diminuent fortement lorsque l'espacement augmente, dans chacune des deux positions étudiées. La répétition a également une influence très significative. Les contraintes sont beaucoup plus faibles dans la répétition 3 où le «Site Index» est plus élevé ( 27 mètres, contre 21 mètres et 20,5 mètres en répétitions 2 et 1 respectivement). La figure 2 a illustre les variations du diamètre tangentiel pour la moyenne des deux positions de prélèvement. (Se rappeler que les contraintes diminuent lorsque TG augmente). Ainsi, dans les peuplements où ils poussent plus vite, soit à cause d'un espacement supérieur, soit à cause d'une meilleure fertilité, les arbres développent moins de contraintes de croissance. Ce résultat est analogue à celui obtenu pour le hêtre (FERRAND, 1982 b), en ce qui concerne l'effet de l'espacement. Mais pas pour la fertilité puisqu'on n'avait trouvé aucune influence du type de station.

Létude des corrélations se montre tout aussi intéressante. Le tableau 5 regroupe les coefficients de corrélation entre les variables technologiques et les caractéristiques du peuplement. Ces dernières n'ont en général été mesurées qu'au niveau moyenne de placette et les corrélations ont donc dû être étudiées à ce niveau (17 degrés de liberté). Seul le diamètre a été mesuré pour chaque arbre, ce qui a permis de calculer les corrélations à deux niveaux supplémentaires : espacement ( 5 degrés de liberté) et arbre dans placette (c'est-à-dire erreur, 162 degrés de liberté).

Légendes de la figure 2

Expérience $n^{\circ} 7$ : Moyennes des observations dans chaque placette :

Influence de la répétition et de l'espacement (ESPA) sur:

a) le diamètre tangentiel des carottes TGM (moyenne des positions 1 et 5);

b) l'infradensité IDM (moyenne des positions 1 et 5 );

c) le retrait tangentiel sans reconditionnement RTM (moyenne des positions $I$ et 5);

d) le retrait radial sans reconditionnement RRM (moyenne des positions 1 et 5).

Experiment no. 7 : Plot mean level:

Influence of replication and spacing (ESPA) on :

a) tangential diameier of the increment cores TGM (mean of upper and opposite side);

b) basic density IDM (mean of upper and opposite side);

c) tangential shrinkage before reconditionning RTM (mean of upper and opposite side);

d) radial shrinkage before reconditionning RRM (mean of upper and opposite side). 


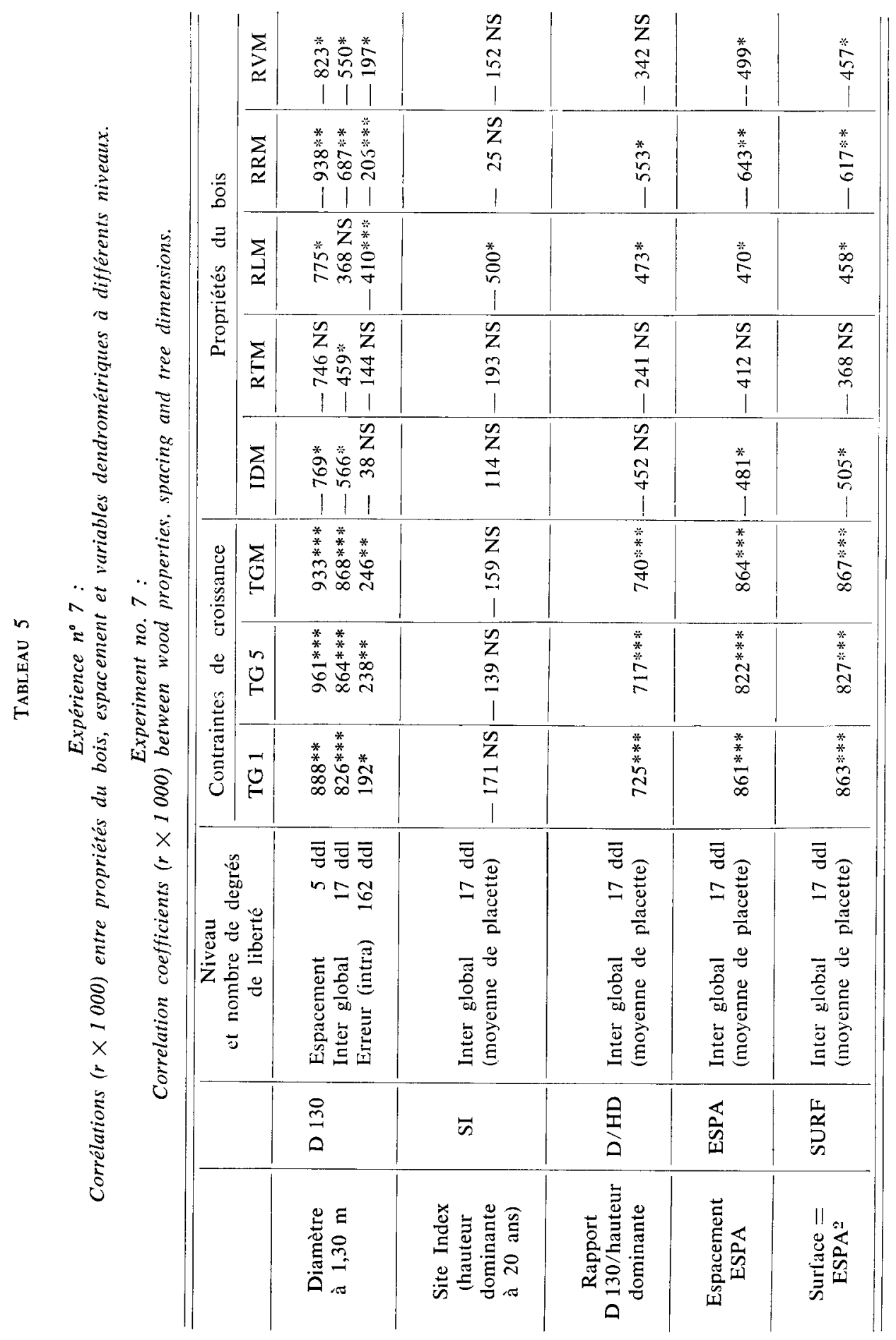


On constate à tous les niveaux une liaison très significative entre le diamètre tangentiel moyen (TGM) et les variables de grosseur des arbres : diamètre, rapport D/HD, espacement, surface disponible par arbre (= carré de l'espacement). Les valeurs de $r$ sont très élevées, comme dans le cas du hêtre et confirment la liaison extrêmement étroite qui a déjà été mise en évidence pour cette dernière espèce entre l'intensité de la compétition et le niveau des contraintes de croissance (1).

En revanche, en corrélation simple, la liaison avec le «Site Index » est non significative. Mais une analyse en régression multiple permet de faire intervenir de manière significative le «Site Index» (voir tableau 6). On arrive à des coefficients de corrélation multiple compris entre 0,90 et 0,94 selon la position autour du tronc, ce qui est exceptionnel entre une variable technologique et des facteurs « externes»: les seules variables espacement (élevé au carré) et "Site Index » (hauteur dominante à 20 ans) expliquent 81 à 88 p. 100 des variations de diamètre tangentiel (estimateur des contraintes de croissance) mesurées dans cette expérience au niveau moyenne de placette.

\section{TABLEAU 6}

Expérience $n^{\circ} 7$ :

Régression multiple : explication du diamètre tangentiel par le carré de l'espacement (SURF) et le «Site Index».

Experiment no. 7 :

Multiple regression analysis : explanation of the tangential diameter by the square of spacing (SURF) and «Site Index》.

\begin{tabular}{|c|c|c|c|c|}
\hline Ligne & Valeur inscrite sur la ligne & TG 1 & TG 5 & TGM \\
\hline (1) & $\begin{array}{l}\text { Coefficient de corrélation simple avec } \\
\text { SURF } \ldots \ldots \ldots \ldots \ldots \ldots \ldots \ldots \ldots\end{array}$ & $0,863 * * *$ & $0.827 * * *$ & $0,867 * * *$ \\
\hline (2) & $\begin{array}{l}\text { Coefficient de corrélation partielle } \\
\text { avec "Site Index» compte tenu } \\
\text { de la ligne (1) } \ldots \ldots \ldots \ldots \ldots \ldots\end{array}$ & $0,667 * *$ & $0,626^{* *}$ & $0,711 * *$ \\
\hline (3) & $\begin{array}{l}\text { Coefficient de corrélation multiple } \\
\text { avec SURF et } \text { "Site Index» } \ldots\end{array}$ & 0,927 & 0,899 & 0,937 \\
\hline & $\begin{array}{l}\text { Test } F \text { à } 2 \text { et } 15 \text { degrés de liberté } \\
\text { correspondant à la ligne (3) ... }\end{array}$ & $45,6 * * *$ & $31,4 * * *$ & $53,8 * * *$ \\
\hline
\end{tabular}

\subsection{Propriétés physiques du bois}

La figure 2 et les tableaux 4 et 5 donnent également les résultats pour les propriétés physiques du bois.

Pour l'infradensité moyenne, ni l'espacement ni la répétition n'ont d'effet notable (tableau 4). Cependant, on note (tableau 5, figure 2 b) une certaine tendance à la

(1) L'examen des deux dernières lignes du tableau 5 montre que l'on peut utiliser indifféremment l'espacement ou la surface disponible par arbre (égale au carré de l'espacement) pour ces calculs de régression linéaire. 
diminution lorsque l'espacement augmente. Cette diminution est faible et de peu de conséquence sur la qualité du bois; elle ne saura constituer un inconvénient si l'on adopte les larges espacements qui semblent être nécessaires à l'amélioration du problème des contraintes de croissance.

D'autre part, on observe d'importantes différences significatives sur les retraits radial et tangentiel, mais leur interprétation est rendue malaisée par la présence d'une forte interaction espacement-répétition. L'examen des corrélations (tableau 5) montre que ces deux retraits ont tendance à diminuer lorsque l'espacement augmente. Il faut pourtant remarquer que dans le modèle mixte, plus aucun test $F$ sur l'espacement ne prend de valeur significative : les différences de retrait liées à l'espacement qui sont observées ici ne permettent donc pas de prédire des différences du même type dans d'autres stations.

En revanche, l'effet de la répétition montre qu'on peut attendre en général une diminution des retraits tangentiel et radial dans les stations plus fertiles figures $2 \mathrm{c}$ et 2 d).

Enfin, le retrait longitudinal (qui est négatif et très faible) est minimum (en valeur absolue) en répétition 1 (la moins fertile) mais minimum aussi pour l'espacement le plus grand. Ce comportement est difficile à expliquer.

En conclusion, on peut retenir que :

-- l'analyse de la variance ne permet de prévoir aucun effet de l'espacement sur les propriétés physiques, même si l'étude des corrélations montre dans le cas particulier de l'expérience $n " 7$ une diminution des valeurs de retrait et dinfradensité aux grands espacements; fertiles.

- les retraits tangentiel et radial sont plus faibles dans les lieux les plus

On peut également remarquer sur la figure 2 (b, c, d) que l'éventail des propriétés physiques est très large à l'espacement $4,88 \mathrm{~m} \times 4,88 \mathrm{~m}$ pour devenir presque nul à l'espacement $6,86 \mathrm{~m} \times 6,86 \mathrm{~m}$ où les cimes des arbres ne sont pas encore en contact.

\section{Résultats des expériences d'éclaircies}

La certitude de l'influence de l'écartement à la plantation sur les contraintes de croissance donne un moyen technique efficace pour diminuer le niveau des contraintes dans les futures plantations. Cependant, pour les forêts existantes et les régénérations à venir, il est important de savoir si une augmentation de l'espacement causée par une éclaircie possède la même efficacité. Là encore, nous avons pu travailler dans les placettes expérimentales de la Forests Commission of Victoria situées à proximité de Melbourne; elles sont décrites dans le tableau 1.

\subsection{Expérience $" 1 " 8$}

L'expérience $\mathrm{n}^{\prime \prime} 8$, située à Clear Hills près de Mansfield concerne un essai assez ancien (coupe d'éclaircie en 1963-1964 à lâge de 6-7 ans), installé en altitude 
en pleine aire naturelle d'E. delegatensis; les densités à l'hectare choisies étaient assez fortes (témoin non éclairci, 1236 et 618 tiges/ha), mais ont été un peu diminuées par la mortalité naturelle (en 1980 il restait en moyenne 2187,877 et 568 tiges/ha).

On a tout d'abord vérifié l'existence de la dissymétrie des contraintes de croissance entre le côté tendu et le côté opposé. En effet, ce peuplement est issu de régénération par semis et non de plantation. Les densités à l'hectare à l'origine étaient donc beaucoup plus élevées qu'à Mont Macedon et on pouvait penser qu'il s'ensuivrait une amélioration de la rectitude et de la répartition des contraintes de croissance autour du tronc. On a donc prélevé des carottes en position 1 et 5 (côté tendu et opposé respectivement) dans 30 arbres (10 par intensité d'éclaircie) de la répétition 1. La différence moyenne de diamètre tangentiel TG 5-TG 1 s'établit à $7,9 \mu \mathrm{m}$ et le test des moyennes appariées (aléa de Gauss) prend la valeur de 3,02 significative au risque 1 p. 100. Par conséquent, la dissymétrie des contraintes existe dans ce peuplement issu de semis tout comme dans la plantation de Mont Macedon. On peut d'ailleurs signaler que tous les arbres sondés dans cette expérience avaient une inclinaison décelable avec le fil à plomb.

Ensuite, on a prélevé dans la deuxième répétition 30 autres carottes (10 par modalité d'éclaircie) du côté tendu uniquement. Le tableau 7 donne les résultats des mesures de diamètre tangentiel, et de l'analyse de la variance dans un modèle à effets fixes.

\section{Tableau 7}

Expérience $n^{\circ} 8$ : Résultats des mesures et analyse de la variance.

Experiment no. 8 : Results of measurements and analysis of variance.

\begin{tabular}{|c|c|c|c|c|c|}
\hline \multicolumn{3}{|c|}{ Diamètre tangentiel côté tendu TG 1} & \multicolumn{3}{|c|}{ Analyse de variance } \\
\hline & $\begin{array}{c}\text { Répétition } \\
1\end{array}$ & $\begin{array}{c}\text { Répétition } \\
2\end{array}$ & Facteur testé & $\begin{array}{l}\text { Degrés } \\
\text { de liberté }\end{array}$ & Test F \\
\hline Témoin & 5072,8 & 5078,1 & \multirow{4}{*}{$\begin{array}{l}\text { Répétition } \ldots \ldots \\
\text { Eclaircie } \ldots \ldots \ldots \\
\text { Interaction } \\
\text { rép./éclaircie .. }\end{array}$} & 1,54 & $7,03 *$ \\
\hline $1236 /$ ha & 5062,2 & 5077,6 & & 2,54 & NS \\
\hline $618 /$ ha & 5074,8 & 5090,9 & & \multirow{2}{*}{2,54} & \multirow{2}{*}{ NS } \\
\hline Moyenne & 5069,9 & 5082,9 & & & \\
\hline
\end{tabular}

A ces densités (qui sont d’autant plus élevées que le peuplement a 24 ans), les éclaircies déjà anciennes qui ont été pratiquées n'ont pas d'influence sur le diamètre tangentiel des carottes (le résultat du test est également non significatif dans un modèle mixte). Il n'y a donc pas lieu de penser qu'elles ont diminué significativement le niveau moyen des contraintes de croissance. Tout au plus peut-on noter que, pour chaque répétition, la valeur maximale de TG se rencontre dans la placette la plus éclaircie.

En revanche, on constate une différence entre les répétitions qui ne peut être attribuée à aucun des facteurs qui ont été mesurés. En effet, les diamètres à $1,30 \mathrm{~m}$ 
et les hauteurs dominantes sont très voisins dans les deux répétitions. Seule une mortalité légèrement plus forte a porté les densités à l'hectare à des valeurs plus faibles en répétition 2 et pourrait constituer un élément d'explication.

\subsection{Expérience $n^{0} 9$}

L'expérience $\mathrm{n}^{\circ} 9$ (à Doweys Spur) n'a été mesurée que faute d'un meilleur matériel. En effet, chaque traitement n'y est répété qu'une fois, ce qui est un inconvénient majeur pour l'analyse statistique. Cependant, elle avait l'intérêt de correspondre à des éclaircies précoces et très intenses dans un peuplement issu de régénération artificielle (par semis aérien).

Dix arbres ont été sondés dans chaque placette du côté tendu. Les résultats sont présentés dans le tableau 8.

\section{TABLEAU 8}

Expérience $n^{\circ} 9$ : Résultats des mesures et analyse de la variance.

Experiment no. 9 : Results of measurements and analysis of variance.

\begin{tabular}{c|c|c|c|c|c}
\hline & \multicolumn{3}{|c|}{ Moyennes par placette } & $\begin{array}{c}\text { Analyse } \\
\text { de la variance }\end{array}$ \\
\hline & Témoin & $\begin{array}{c}618 \\
\text { tiges/ha }\end{array}$ & $\begin{array}{c}370 \\
\text { tiges/ha }\end{array}$ & $\begin{array}{c}123 \\
\text { tiges/ha }\end{array}$ & $\begin{array}{c}\text { Test } \mathrm{F} \\
\text { ì } 3 \text { et } 36 \text { degrés } \\
\text { de liberté }\end{array}$ \\
\hline $\begin{array}{c}\text { Diamètre } \\
\text { à } 1,30 \mathrm{~m} \\
\mathrm{D} 130 \mathrm{en} \mathrm{mm}\end{array}$ & 208 & 262 & 265 & 336 & $\mathrm{~F}=32,5^{* *}$ \\
\hline $\begin{array}{c}\text { Diamètre } \\
\text { tangentiel } \\
\text { des carottes TG 1 } \\
\text { en } \mu \mathrm{m}\end{array}$ & 5084,4 & 5095,5 & 5086,0 & 5102,9 & $\mathrm{~F}=3,35^{*}$ \\
\hline
\end{tabular}

Il y a un effet significatif du traitement sur TG 1. L'écart entre le témoin et l'éclaircie la plus forte est important. Les autres placettes se classent entre ces deux extrêmes, mais pas dans l'ordre où on les attendait. Il y a certainement un phénomène parasite dans la placette à 370 tiges/ga ; TG 1 y est plus faible que dans la placette à 618 tiges/ha, mais surtout le diamètre D 130 y est le même, ce qui n'est pas normal du tout. Si l'on s'intéresse aux hauteurs des arbres, on s'aperçoit de même que la hauteur dominante était en 1979 de $20,5 \mathrm{~m}$ dans la placette à 370 tiges/ha, contre $21,8 \mathrm{~m}$ dans l'autre placette. En 1981, la même différence de 1,3 m subsiste et est significative au risque 5 p. 100 . On peut donc soupçonner une hétérogénéité du milieu, qui s'accompagnerait comme à Mont Macedon de contraintes de croissance plus fortes dans le site le moins fertile. Cependant, on ne peut que regretter l'absence de répétition qui empêche une analyse plus approfondie. 
D'autre part, comme à Mont Macedon, la corrélation entre TG 1 et D 130 est significative $\left(r=0,88^{*}\right.$ au niveau inter, c'est-à-dire entre moyennes de placettes; $r=0,39^{*}$ au niveau intra, 36 degrés de liberté). Il faut cependant se rappeler que dans ces peuplements équiennes le diamètre à $1,30 \mathrm{~m}$ est surtout un indicateur de vitesse de croissance (quelle que soit son origine, génétique, sylvicole, ou due au milieu), au contraire des peuplements naturels inéquiennes où il indique aussi l'âge. De plus, au niveau inter, le coefficient de corrélation partielle de TG 1 avec la hauteur dominante (une fois prise en compte la corrélation simple entre TG 1 et $\mathrm{D} 130$ ) vaut $0,997^{*}$, ce qui est une autre façon d'exprimer l'influence du milieu que l'on vient de discuter à propos de la placette à 618 tiges/ha.

En conclusion, malgré le handicap du manque de répétitions, il apparaît des résultats très analogues à ceux de l'essai d'espacement : les contraintes sont plus faibles après les fortes éclaircies et plus fortes dans un milieu moins «fertile».

\subsection{Expérience $n^{\circ} 10$ à Bunyip Rd}

\subsection{Présentation}

Au contraire des expériences précédentes qui concernaient toutes Eucalyptus delegatensis, l'expérience $\mathrm{n}^{\circ} 10$ concerne Eucalyptus nitens. (Faute de temps on n'a pu tester la corrélation entre TG et $\varepsilon_{\mathrm{I}}$ sur cette espèce. Il n'y a cependant pas de raison de penser qu'elle puisse donner lieu à des phénomènes fondamentalement différents de ceux qu'on observe avec $E$. delegatensis, le hêtre ou le peuplier). Cette espèce possède une extension géographique très vaste mais ne forme que de petits peuplements enclavés entre ceux d'E. regnans et $E$. delegatensis c'est-à-dire à une altitude assez élevée, où ils bénéficient d'une bonne pluviosité. L'intérêt majeur d'E. nitens (appelé «Shining gum» dans le Victoria) est sa vigueur. Il s'adapte très bien en reboisement à des altitudes très inférieures à celles de son aire d'origine et semble être dans de nombreux pays tempérés l'eucalyptus le plus productif. A titre d'exemple, citons la hauteur dominante à 12 ans dans les placettes non éclaircies de l'expérience $\mathrm{n}^{\circ} 10$, qui est supérieure à 25 mètres. Son bois a été peu utilisé jusqu'à présent en Australie. Il est très blanc, semble posséder des propriétés papetières intéressantes et peut être utilisé comme bois d'œuvre. Cependant, sa valeur n'est pas considérée comme égale à celle du bois d'E. delegatensis qui est un des bois d'œuvre les plus employés du sud de l'Australie et qui fournit même des placages tranchés très décoratifs (couleur rose pâle) qui sont parfois ondés.

L'expérience $n^{\circ} 10$ est installée (comme la $n^{\circ}$ 9) sur les hauteurs qui dominent la vallée de la Yarra River à Powelltown près de Melbourne. Le peuplement a été régénéré par semis aérien d'un mélange de graines d'E. regnans, $E$. delegatensis et $E$. nitens. Mais aujourd'hui, la majorité des arbres dominants est de l'espèce nitens. Une éclaircie intense a été pratiquée à l'âge de 5 ans, avec deux répétitions de 3 modalités : témoin non éclairci, conservation de 370 tiges/ha et 123 tiges/ha. On a prélevé une carotte par arbre du côté tendu, dans 10 arbres par parcelle unitaire, soit 60 carottes.

\subsection{Résultats}

Le tableau 9 donne les résultats des mesures et ceux de l'analyse de la variance. (Cette dernière n'a pas été réalisée sur les variables hauteur dominante et espacement 


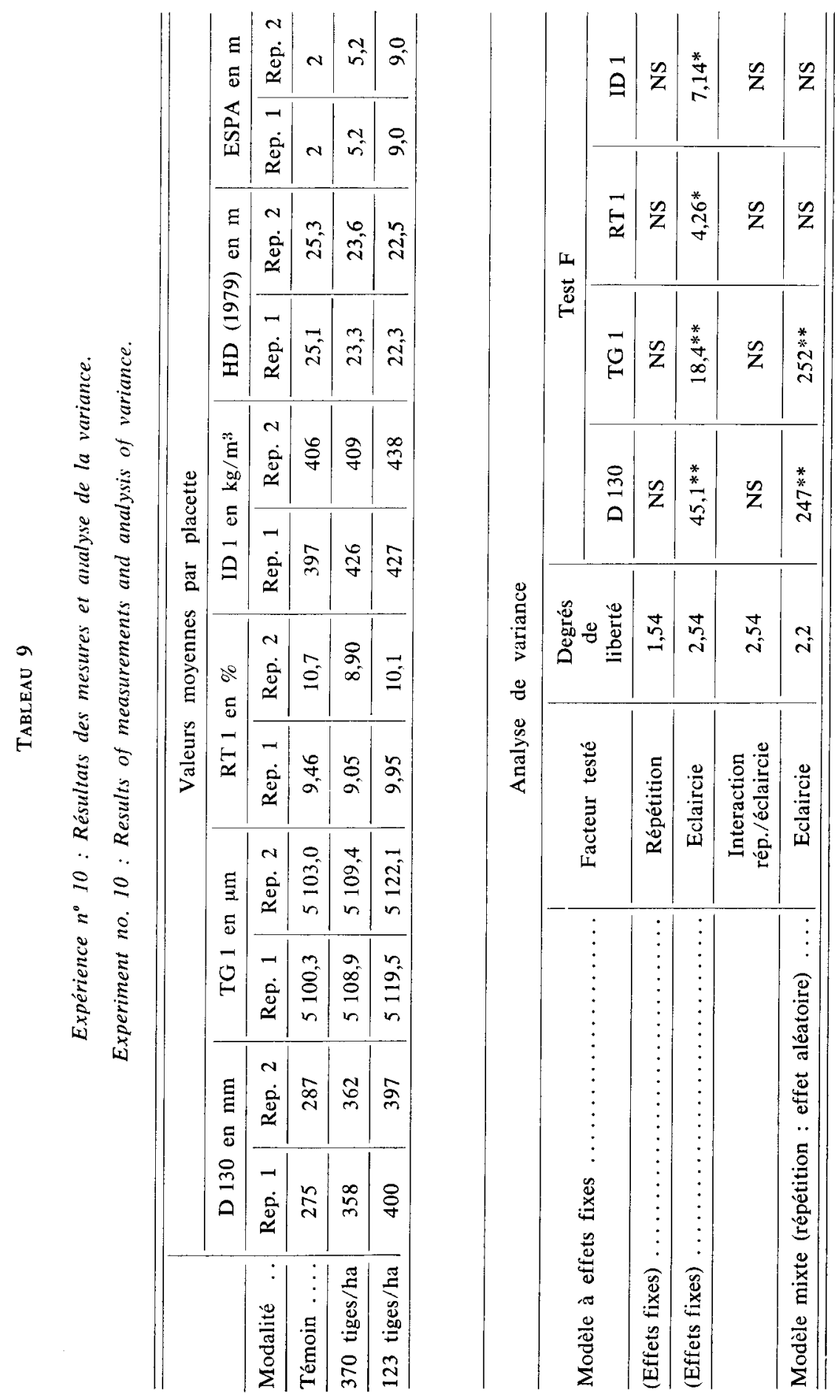


moyen qui ne sont connues qu'au niveau moyenne de placette). De plus, la valeur de l'espacement dans les placettes témoin est une estimation et n'a pas fait l'objet d'une mesure précise, de même que la hauteur dominante dans une de ces placettes. Comme pour l'expérience $\mathrm{n}^{\circ} 7$, les tests statistiques ont été réalisés de deux façons différentes selon que l'effet de la répétition (donc de la station) était considéré comme fixe ou aléatoire.

On ne constate aucun effet de la répétition sur les variables mesurées. En revanche, elles sont toutes influencées par l'éclaircie dans le cas de l'hypothèse d'effets fixes du facteur répétition. Le diamètre tangentiel des carottes augmente beaucoup (donc les contraintes diminuent) lorsque la densité à l'hectare diminue. L'infradensité augmente un peu, et le retrait tangentiel est minimum à 370 tiges/ha. Il y a une similitude parfaite avec l'essai d'espacement d'E. delegatensis pour les contraintes de croissance et une certaine ressemblance pour le retrait tangentiel. En revanche, l'infradensité a un comportement opposé.

Lorsqu'on réalise l'analyse selon un modèle mixte, les résultats demeurent les mêmes pour le diamètre à $1,30 \mathrm{~m}$ et les contraintes de croissance. En revanche, il n'y a plus d'effet de l'éclaircie sur le retrait tangentiel ni l'infradensité. Cela signifie, comme dans l'expérience $\mathrm{n}^{\prime \prime} 7$, que pour ces variables les résultats ne permettent pas de généraliser les différences observées à l'intérieur du dispositif à d'autres stations du même type.

Tableau 10

Expérience $n^{\prime \prime} 10$ :

Corrélations $(r \times 1000)$ au niveau moyenne de placette (4 degrés de liberté).

Experiment no. 10 :

Correlation coefficients $(r \times 1000)$ at «plot mean» level (4 degrees of freedom).

\begin{tabular}{|c|c|c|c|c|c|c|}
\hline & & D 130 & TG 1 & RT 1 & ID 1 & HD \\
\hline $\begin{array}{l}\text { Diamètre tangentiel } \\
\text { des carottes ...... }\end{array}$ & TG 1 & $944 * *$ & & & & \\
\hline Retrait tangentiel .. & RT 1 & $-148 \mathrm{NS}$ & $125 \mathrm{NS}$ & & & \\
\hline Infradensité & ID 1 & $881 *$ & $910^{*}$ & $94 \mathrm{NS}$ & & \\
\hline Hauteur dominante & $\mathrm{HD}$ & $-986^{* * *}$ & $-939 * *$ & $178 \mathrm{NS}$ & $-890^{*}$ & \\
\hline Espacement moyen & ESPA & $967 * *$ & $988^{* * *}$ & $7 \mathrm{NS}$ & $893 *$ & $-976^{* * *}$ \\
\hline
\end{tabular}

On peut examiner encore les corrélations entre les variables au niveau moyenne de placette (tableau 10). La corrélation entre TG 1 et l'espacement (ESPA) est significative au risque 1 p. $1000\left(\mathrm{r}=0,988^{* * *}\right)$ et représentée sur la figure 3 . De plus, la corrélation partielle avec la hauteur dominante (HD) est significative au risque 5 p. $100\left(\mathrm{r}=0,950^{*}\right)$. Pour l'explication de TG 1 par ESPA et HD, cela porte le coefficient de corrélation multiple à $R=0,9988$ (test attaché à cette valeur de 
$\left.\mathbf{R}: \mathbf{F}(2,3)=651^{* * *}\right)$; bien que reposant sur un faible nombre de données, une telle liaison entre une caractéristique technologique et des facteurs externes mérite d'être soulignée.

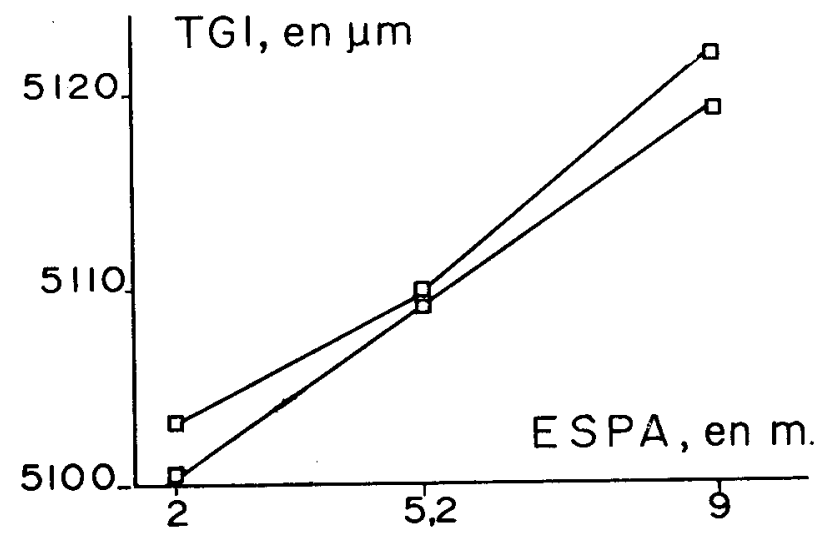

FIG. 3

Expérience $n^{\circ} 10$ : Moyenne des observations dans chaque placette : Influence de l'espacement (ESPA) sur le diamètre tangentiel des carottes TG 1 (côté tendu).

Experiment $n^{\circ} 10:$ Plot mean level : influence of spacing (ESPA) on the tangential diameter of the increment cores TG 1 (upper side).

Comme dans les expériences précédentes on observe de fortes liaisons entre le diamètre $\mathrm{D} 130$ et la qualité du bois, mais le diamètre des arbres ne doit être considéré que comme un résultat de la sylviculture. De plus, la diminution de hauteur dominante associée aux fortes éclaircies dans les placettes à 370 et 123 tiges à l'hectare est un résultat peu attendu. Cette relation mériterait d'être étudiée plus à fond dans des dispositifs où le milieu serait mieux connu.

Enfin, on relève au niveau «intra» deux corrélations significatives au risque 5 p. 100 entre le diamètre tangentiel des carottes (TG 1) et l'infradensité, comme au niveau inter, et entre TG 1 et le retrait tangentiel.

En conclusion, le niveau moyen des contraintes de croissance est dans cette expérience sous la dépendance presque complète de l'intensité de l'éclaircie et de la hauteur dominante.

\section{Discussion}

A l'issue de ces expériences, il se dégage deux conclusions très claires :

- au même âge, plus l'espacement est grand, plus les contraintes sont faibles ; faibles.

- une forte hauteur dominante s'accompagne de contraintes légèrement plus 
On peut même développer une notion de seuil d'espacement (ou de densité à l'hectare) puisque les éclaircies insuffisantes de l'expérience $\mathrm{n}^{\circ} 8$ ne se sont accompagnées d'aucune diminution des contraintes. De plus, l'examen attentif de la figure 2 montre qu'à Mont Macedon les trois espacements les plus faibles présentent des niveaux de contraintes uniformément élevés dans chacune des trois répétitions. La valeur de 'TGM augmente ensuite graduellement jusqu'aux espacements les plus grands. On constate même que chaque placette à $2,44 \mathrm{~m} \times 2,44 \mathrm{~m}$ donne une valeur de TGM plus faible (donc contraintes plus fortes) que celle à l'espacement $1,71 \mathrm{~m} \times 1,71 \mathrm{~m}$ dans la même répétition. Cela s'inscrit parfaitement dans le cadre de l'hypothèse que nous avons déjà formulée à propos du hêtre : le niveau moyen des contraintes dans une placette augmente fortement avec la compétition entre les arbres. En effet, les eucalyptus supportent mal la compétition et les peuplements sont le lieu d'une mortalité très importante en l'absence d'intervention sylvicole : on se retrouve rapidement en présence d'un peuplement beaucoup moins dense où quelques gros arbres ont éliminé leurs voisins. On peut penser d'après l'aspect des placettes que celles à $1,22 \mathrm{~m} \times 1,22 \mathrm{~m}$ et $1,71 \mathrm{~m} \times 1,71 \mathrm{~m}$ ont déjà dépassé ce stade, tandis que la compétition est encore une lutte «à mort » dans les placettes à $2,44 \mathrm{~m} \times 2,44 \mathrm{~m}$. On constate de même dans l'expérience $n^{\circ} 8$ (bien que les différences soient non significatives), que les valeurs de contraintes les plus élevées se rencontrent non pas dans les placettes non éclaircies, mais dans celles ayant subi l'éclaircie la plus faible.

Ces résultats soulignent donc la nécessité de très grands espacements afin dassurer une croissance très rapide qui s'accompagne de faibles contraintes de croissance. L'évidence expérimentale va ainsi contre une idée très répandue selon laquelle chez les eucalyptus, la croissance rapide est synonyme de fortes contraintes. Cette opinion est fondée principalement sur l'observation de peuplements artificiels (reboisements, «regrowth forest», etc.) et assez jeunes, que l'on compare éventuellement aux vieilles parcelles de la forêt naturelle d'eucalyptus. Ces jeunes peuplements sont le lieu d'une compétition intense ; c'est cela — et non leur croissance rapide - qui est à l'origine des contraintes élevées qu'on y constate. D’ailleurs, les chercheurs néo-zélandais (ReVell, communication personnelle) ont bien constaté en observant le sciage des grumes d'eucalyptus que :

a) au même âge les plus gros arbres sont plus faciles à scier ;

b) les plus difficiles viennent des parcelles non éclaircies;

c) les plus faciles sont les arbres isolés (bords de route, prairie, etc.) ;

d) même les gros arbres dominants des parcelles à fortes contraintes sont difficiles à scier.

Toutes ces impressions sont confirmées en détail par nos mesures, y compris l'assertion d (rappelons que nous n'avons sondé que des arbres dominants, dans toutes les expériences).

Cependant, s'il est clair que l'accélération de croissance d'origine externe (sylviculture ou station) s'accompagne de contraintes plus faibles, on ne sait presque rien de l'influence des causes internes (génétiques) sur les contraintes de croissance et ce point doit encore être étudié.

Il faut remarquer également que l'on a entre les mains toute la méthodologie nécessaire à la définition d'une sylviculture optimale des espèces concernées. En effet, des essais de plantation et d'éclaircie en plus grand nombre permettraient de 
recueillir toutes les données dendrométriques nécessaires à la confection de tables de production dans lesquelles on trouverait, pour chaque catégorie de hauteur dominante à 20 ans ("Site Index»), les densités à l'hectare, circonférences, hauteurs et volumes aux différentes époques de la vie du peuplement. Or on a vu que, à âge constant, le niveau moyen des contraintes de croissance est sous dépendance presque complète de la densité à l'hectare et de la hauteur dominante. Par conséquent, un calcul simple permettrait de compléter ces tables par une valeur moyenne des contraintes pour les arbres enlevés à chaque éclaircie et lors de la récolte finale. Ces tables seraient un outil précieux d'aménagement et de gestion des forêts d'eucalyptus. Cette démarche ne serait pas difficile à mettre sur pied puisqu'il s'agit d'espèces à cultiver à une rotation très courte et avec un faible nombre d'éclaircies (une seule dans de nombreux cas). La difficulté sera surtout de choisir entre : semis suivi d'une éclaircie (précoce donc non commerciale) d'une part, et plantation à large espacement (avec ou sans éclaircie commerciale) d'autre part. La forme et la qualité (élagage, kino (1)) des tiges sera un facteur clé de ce choix, qu'il n'est pas possible de présager, bien qu'il semble que les plantations à très larges espacements non suivies d'opérations de formation des tiges ne puissent pas convenir.

On doit souligner enfin que l'adoption de telles règles de sylviculture plus énergiques diminuera les problèmes d'utilisation du bois liés aux contraintes de croissance non seulement en diminuant le niveau moyen des contraintes à la périphérie des troncs, mais encore parce que le diamètre moyen des arbres récoltés sera plus fort. En effet, les difficultés rencontrées par les bûcherons et scieurs sont dues au gradient cour-écorce de la contrainte longitudinale plus qu'à cette contrainte ellemême. Or, même à contrainte égale à la périphérie du tronc, plus le diamètre d'un arbre est gros, plus le gradient de la contrainte est faible. Par conséquent, une sylviculture produisant des arbres plus gros et à plus faible contrainte longitudinale diminuera doublement les problèmes liés aux contraintes de croissance.

Pour réaliser une telle sylviculture, les gestionnaires pourront tant qu'ils ne disposeront pas de données plus précises, utiliser comme guides des facteurs simples et synthétiques tels que le rapport diamètre sur hauteur dominante ou le facteur de Hart-Becking (espacement/hauteur dominante).

Pour réduire de manière importante le niveau des contraintes de croissance sans tomber en dessous d'un certain seuil de densité à l'hectare on peut estimer comme raisonnables des valeurs d'environ 1,4 p. 100 pour le rapport diamètre/hauteur dominante et 29 p. 100 pour le facteur de Hart-Becking, dans le cas d'Eucalyptus delegatensis et E. riltens. Ces valeurs sont plus élevées que celles que l'on pratique habituellemerıt; cela correspond à la nécessité de diminuer la compétition entre les arbres pour diminuer les contraintes de croissance.

Reçu pour publication en décembre 1981.

(1) Les veines de kino sont un grave défaut du bois d'eucalyptus et l'on sait que leur présence est très liée aux conditions du milieu. 


\section{Remerciements}

Cette étude a été réalisée grâce au support financier d'une bourse de l'O.T.A.N. Mes chaleureux remerciements vont au docteur W.E. Hillis (Division of Building Research, C.S.I.R.O., Melbourne, Vic., Australia) qui m'a accueilli dans son laboratoire à Melbourne, W.D. InCOLL (Research Officer, Forests Commission of Victoria) qui m'a donné accès aux plantations expérimentales, ainsi qu'à H. POLGE et aux membres de la Station de Recherches sur la Qualité des Bois (C.N.R.F., Champenoux), qui m’ont apporté leur aide.

\section{Summary}

Study of growth stresses. 3rd Part : Eucalyptus delegatensis and E. nitens :

\section{Influence of silviculture and site index}

About 550 growth strain measurements have been performed on Eucalyptus delegatensis and Eucalyptus nitens in spacing and thinning trials located near Melbourne (Victoria, Australia). Results showed that at the same age, the larger the spacing was the smaller the growth strain. Therefore silviculture appears as a very efficient way to reduce conversion problems due to growth stresses. Growth strain decreased too as increased the mean dominant height. Thus, growth strain is influenced by the site.

These results show that it is possible to predict which growth strain level will appear as a consequence of a given silviculture in a given site.

\section{Références bibliographiques}

BARr N.A., 1980. - Sawing qualities of Eucalyptus saligna Sm. as a guide to selection of trees for breeding and seed collection. I.U.F.R.O. Symposium and Workshop, Aguas de Sao Pedro, Sao Paulo, Brazil, 6 p.

Dagnelie P., 1975. Théories et méthodes statistiques, vol. 2. Presses Agronomiques de Gembloux.

Ferrand J.Ch., 1982 a. Etude des contraintes de croissance. 1 - Méthode de mesure sur carottes de sondage. Ann. Sci. for., 39 (2), 109-142.

FERrand J.Ch., 1982 b. Etude des contraintes de croissance. 2 - Variabilité en forêt des contraintes de croissance du hêtre. Ann. Sci. for., 39 (3), 187-218.

Mc KIMm R.J., 1981. Communication personnelle.

Nicholson J.E., 1971. A rapid method for estimating longitudinal growth stresses in logs. Wood Science and Technlogy, vol. 5, 40-48.

RevelL D.H., 1981. Communication personnelle.

WAUGH G., 1977. Variation in selected growth and wood characteristics in plantation grown Eucalyptus regnans. Paper 18th Forest Products Conf. Melbourne. 